\title{
Effects of multiple experimenters on attachment behavior of mallard ducklings (Anas platyrhynchos platyrhynchos)
}

\author{
CORY JOHN LINDGREN, ANGIE LOMBARDI, TERRY J. BUSS, and L. JAMES SHAPIRO \\ University of Manitoba, Winnipeg, Manitoba, Canada
}

\begin{abstract}
The purpose of this study was to determine if multiple experimenters would have any effect on the preferences of mallard ducklings for broods of 3,6 , or 9 ducklings. Three different experimenters ran 2 different subjects each day for 7 consecutive days. The mean age of the subjects was $24 \mathrm{~h}$ at the onset of testing. The dependent variable was the amount of time each subject spent with a brood of 3,6 , or 9 ducklings during a 15 -min trial. It was found that a brood of 9 ducklings was preferred significantly more often $(p<.05)$ than a brood of 6 or a brood of 3 ducklings. The results also indicated that with the task described and the ages involved, different experimenters did not contribute significant sources of variation to the results obtained.
\end{abstract}

Developmental research involving avian species can potentially consume large amounts of one experimenter's time and energy. The use of multiple experimenters can ease the burden that would be placed upon one experimenter in terms of the time and the energy required for a particular study.

The Avian Behaviour Laboratory in the Department of Psychology at the University of Manitoba is interested in those mechanisms that mediate an attachment between parents and their young, between young and their parents, and among the young. For mallard ducklings (Anas platyrhynchos platyrhynchos) and giant Canada goose goslings (Branta canadensis maxima), the developmental period of interest to us is the first 7 days of the young bird's life. Shapiro (1977) stated that the formation of an attachment is a continous phenomenon and does not end when a subject reaches 24 or $36 \mathrm{~h}$ of age.

Over a 7-day testing period, an experimenter is subject to many possible disruptions. The experimenter may become ill, grow tired, be injured, have a death in the family, or be subject to an array of unexpected and unanticipated events.

The use of multiple experimenters would minimize the problems caused by such disruptions. Over a 7-day testing period, two or three trained experimenters can divide the time required by the experiment equally among themselves. If one experimenter becomes subject to an unforeseen event, another experimenter can replace the stricken experimenter and continue the research that may have taken months to prepare. The avian literature illustrates the formation of research teams that ranged in size from two experimenters (Kertzman \& Demarest, 1982; Taylor \& Sluckin, 1964) to four experimenters (Taylor, Sluckin, Hewitt, \& Guiton, 1967) to five experimenters (Entrikin, Swanson, Weidoff, Patterson, \& Wilson,

Requests for reprints should be sent to L. James Shapiro, Avian Behaviour Laboratory, Department of Psychology, University of Manitoba, Winnipeg, MB R3T 2N2, Canada.
1977). These experiments, however, do not state whether one or more than one experimenter ran the subjects and, if the latter, whether there was an experimenter effect.

The use of multiple experimenters introduces a new source of variability to the study under investigation. The possible effects of multiple experimenters and the possibility of an interaction between this variable and other independent variables being used in the experiment must be evaluated. The purpose of this article is to examine the possible effects of multiple experimenters on avian attachment behavior. We hypothesized that the use of three different experimenters to obtain data should not affect the behavior in question. A study involving brood preferrences that was initially done by one experimenter (Shapiro, 1980) was repeated using three experimenters. In the original study, a brood of 9 ducklings was found to be preferred significantly more than broods of 6 or 3 ducklings.

\section{METHOD}

\section{Subjects and Apparatus}

The subjects were 42 mallard ducklings hatched from eggs laid by 50 adult mallards (10 males and 40 females) and housed in the Avian Behaviour Laboratory in the Department of Psychology at the University of Manitoba. This flock was kept on a reversed season lighting schedule (i.e., during the winter months, this flock was on a spring lighting cycle). This flock was part of a larger, wild flock maintained at the Field Station of the Avian Behaviour Laboratory at Glenlea, Manitoba.

The eggs were collected from the adults daily, washed, and refrigerated at $12.8^{\circ} \mathrm{C}$. No eggs were refrigerated for more than 1 week. After being disinfected with Phisohex, the eggs were set in a Petersime (300 North Bridge Street, Gettysburg, OH 45328) Model 1 incubator at a temperature of $37.5^{\circ} \mathrm{C}$ for 24 days. On Day 24 of incubation, the eggs were placed in a Petersime H145 hatcher.

Upon hatching, the experimental subjects were randomly placed into one of seven compartments of a Petersime Model 2S-D brood unit. Six experimental subjects were randomly assigned to each compartment. A total of 42 subjects were assigned to compartments. One subject died on Day 7.

The subjects were able to regulate their ambient temperature by moving toward or away from a centralized heat source. The temperature of the 
brood unit compartments was $35^{\circ} \mathrm{C}$ for the first 3 days. It was then reduced $1.5^{\circ} \mathrm{C}$ per day to $29^{\circ} \mathrm{C}$. The temperature of the room that contained the brood unit was $23^{\circ} \mathrm{C}$. Chick starter with a $21 \%$ protein content and water (in separate troughs) were constantly available to all the subjects in their separate compartments.

Eighteen ducklings were used as stimulus objects in this experiment. These ducklings had a mean age of $24 \mathrm{~h}$ at the onset of this study. All stimulus objects were housed in one compartment of a Petersime Model 2S-D brood unit. On each day, the stimulus objects were transported to an experimental chamber, where each was randomly assigned to a brood of 3, 6, or 9 ducklings. The broods were randomly assigned to one of four circular wire-mesh pens that were placed in each corner of a $1.54 \mathrm{~m}^{2}$ sand-covered table. The circular pens were made of 2.54 $\mathrm{cm}^{2}$ wire mesh and were $42 \mathrm{~cm}$ in diameter and $41 \mathrm{~cm}$ high. The sandcovered table was inside a $2.44 \times 2.44 \times 2.0 \mathrm{~m}$ (internal dimensions) sound-deadened experimental chamber. One of the circular pens remained empty on each trial. After each trial, the composition of each brood was rearranged, so that no brood contained exactly the same stimulus objects on any given trial. Hatching and brooding procedures were similar to those used with the experimental subjects. Upon hatching, the stimulus objects were kept apart from the experimental subjects, but both groups were housed in the same brood unit. For a complete description of the apparatus used in this experiment, the reader is referred to Shapiro $(1970,1978)$.

\section{Procedure}

Each of the 3 experimenters ran 2 subjects on each of the 7 testing days, except on Day 7 when 1 experimenter ran only 1 subject. This situation resulted because 1 experimental subject died on Day 7. The mean of the other 5 subjects used on Day 7 was substituted for Subject 6 for statistical purposes. On each testing day, the order in which the experimenters ran their subjects was randomly determined. The position of each of the three broods was randomly selected for each trial in order to control for any position preferences.

Each experimental subject was transported to the experimental chamber inside a $16.5 \times 35.5 \times 15.2 \mathrm{~cm}$ opaque transport box and was placed at the center of the testing platform, in the dark, facing the empty quadrant. The experimenter then placed himself/herself in front of a oneway vision window and simultaneously turned on the lights inside the experimental chamber and automatically activated a four-quadrant timer and latency clock. The four-quadrant timer and latency clock was used by the experimenter to record the amount of time spent in each quadrant by an experimental subject. After each 15-min trial, the four-quadrant timer and latency clock automatically turned off the lights inside the experimental chamber, and the trial was ended. The experimental subject was then returned to its brood unit compartment and identified with a metal bandette to ensure that it was not used again in this experiment. Hence, with the exception of Day 7, all experimental subjects were exposed to 5 "brood mates" while the 6th was being tested.

\section{RESULTS}

The data were analyzed using a three-way analysis of variance with repeated measures on one factor. Brood size was the repeated measures factor, and the between-group factors were the age of the subject (days) and the experimenter. A significant main effect was obtained for the brood-size factor $[F(3,24)=53.13, p<.0001]$. This result is similar to the results reported by Shapiro (1980). No significant main effect was obtained for the experimenter factor or the days factor. There were no significant interactions.

The Newman-Keuls test performed on the means associated with the stimulus objects factor showed that a brood of 9 was preferred significantly more often $(p<.05)$ than a brood of 6 , a brood of 3 , and the empty quadrant. The brood of 6 was preferred significantly more often $(p<.05)$ than the brood of 3 and the empty quad-
Table 1

Mean Time (in min), Collapsed over Days, Spent with Each Brood by Subjects Run By Each Experimenter

\begin{tabular}{lcrrrr}
\hline & \multicolumn{5}{c}{ Brood Size } \\
\cline { 2 - 5 } Experimenter & 0 & 3 & \multicolumn{1}{c}{6} & \multicolumn{1}{c}{9} & Total \\
\hline A.L. & 0.12 & 2.55 & 3.52 & 8.53 & 14.72 \\
T.B. & 0.11 & 3.01 & 4.85 & 6.94 & 14.91 \\
C.L. & 0.05 & 1.05 & 4.61 & 9.07 & 14.78 \\
Total & 0.28 & 6.61 & 12.98 & 24.54 & 44.41 \\
\hline
\end{tabular}

rant, and the brood of 3 was preferred significantly more often $(p<.05)$ than the empty quadrant.

The mean time, collapsed over days, spent in each quadrant by the experimental subject run by each experimenter is presented in Table 1.

\section{DISCUSSION}

The results indicate that with the task described and the ages involved, different experimenters did not contribute significant sources of variation to the results obtained.

When working with newly hatched organisms, the researcher must be able to begin experimenting when the organism itself decides to commence life outside the shell. In natural settings, mallards and giant Canada geese provide this opportunity once a year. If the experimenter is not ready to begin data collection at this time, an entire field season may be lost and the associated months of preparation will be worth very little. Under laboratory conditions, however, birds can be artificially induced to lay eggs when no production would otherwise result. Experimenters can then utilize more than one egg-laying season. With multiple experimenters, the probabilities are increased that when the organism decides to hatch, at least one experimenter will be ready to begin experimentation.

When using multiple experimenters to collect data, it is important that they are trained sufficiently and that variation in data collection between them is minimized. It must be demonstrated that variation between experimenters will not affect the outcome of the experiment. This study demonstrated that three particular experimenters did not affect the outcome of an experiment of a particular type.

\section{REFERENCES}

Entrikin, R. K., Swanson, K. L., Weidoff, P. M., Patterson, G. T., \& Wilson, B. W. (1977). Avian muscular dystrophy: Functional and biochemical improvement with diphenylhydantoin. Science, 195, 873-875.

Kertzman, C., \& Demarest, J. (1982). Irreversibility of imprinting after active vs. passive exposure to the object. Journal of Comparative \& Physiological Psychology, 96, 130-142.

SHAPIRO, L. J. (1970). Automation and experimental control in a laboratory for imprinting research. Journal of Comparative \& Physiological Psychology, 73, 421-426.

SHAPIRo, L. J. (1977). Developing preferences for live female models of the same or other species in white peking ducklings. Animal Behaviour, 25, 849-858.

ShapIRo, L. J. (1978). The Avian Behaviour Laboratory: A laboratory for waterfowl research. Journal of Biological Psychology, 20, 2-10.

SHAPIRo, L. J. (1980). Species identification in birds: A review and synthesis. In A. Roy (Ed.), Species identity and attachment: $A$ phylogenetic evaluation (pp. 69-111). New York: Garland STPM Press.

Taylor, A., Sluckin, W., Hewitt, R., \& Guiton, P. (1967). The formation of attachments by domestic chicks to two textures. Animal Behaviour, 15, 514-519.

TAYLOR, K. F., \& SLUCKIN, W. (1964). Flocking of domestic chicks. Nature, 201, 108-109.

(Manuscript received for publication October 5, 1987.) 